\title{
The synthesis of condensed imidazoles II. A simple synthesis of some 1,5-diaryl-3-[2-(naphtho[2,3-d]imidazol-2-yl)]formazans and its derivatives ${ }^{1}$
}

\author{
Iveta Fryšová ", Jan Slouka, and Jan Hlaváč \\ Department of Organic Chemistry, Palacký University, Tř. Svobody 8, 77146 Olomouc, \\ Czech Republic \\ E-mail:frysova@orgchem.upol.cz
}

\begin{abstract}
The condensation reaction of 1,5-diaryl-3-formazylglyoxylic acids (1) with 2,3diaminonaphtalene affords 1,5-diaryl-3-[2-(naphtho[2,3-d]imidazol-2-yl)]-formazans (2) which have been transformed by reductive splitting into $\mathrm{N}^{\prime}$-aryl-naphtho[2,3- $d$ ]-imidazole-2carbohydrazonamide (3). Oxidative cyclization of formazans (2) leads to the 2,3-diaryl-5(naphtho[2,3-d]imidazol-2-yl)-tetrazolium chlorides (4) and corresponding picrates (5) have been also prepared.
\end{abstract}

Keywords: Formazylglyoxylic acid, 2,3-diaminonaphtalene, formazan

\section{Introduction}

We were interested in course of reactions with other vicinal diamines of non-benzene series. At first we chosed naphtyldiamines where the character of functional groups is a little bit different than at benzenediamines. We have found that 2,3-diaminonaphtalene reacts in same course like 1,2-diaminobenzene. This is advantageous for preparation of naphto[2,3-d]imidazole derivatives which are unknown.

The condensation reaction of $\alpha$-ketocarboxylic acids with 1,2-diaminobenzene which leads to 1,2-dihydroquinoxaline-2-ones has been known for a long time ${ }^{2}$. It is a general method which proceeds with high yields. A large number of substituted quinoxaline derivatives ${ }^{3-5}$ has been prepared in this way. We found that the course of reaction of 1,2-diaminobenzene with 1,5diaryl-3-formazylglyoxylic acids proceeds in a different way and unexpected 1,5-diaryl-3(benzimidazol-2-yl)-formazans are obtained instead of quinoxaline derivatives ${ }^{6,7}$. Herein we focused on preparation of new group of 1,5-diaryl-3-[naphtho[2,3-d]imidazol-2-yl]-formazans (2) for which it was possible to expect two different courses of oxidative cyclization to the corresponding tetrazolium salts (4) or oxidative cyclization, which could lead to the isomeric 
derivatives of [1,2,3]triazolo[1,5-a]naphtho[2,3-d]imidazolinium salts (6). The compounds (2) were also transformed by reductive splitting into $\mathrm{N}^{\prime}$-aryl-naphtho[2,3- $d$ ]-imidazole-2carbohydrazonamide (3). It is interesting, that in this case reaction differs from analogous reaction proceeding at formazans of sacharide family, the result of which are appropriate thiohydrazides ${ }^{8}$.

\section{Results and Discussion}

The condensation reaction of acids (1a-1f) with 2,3-diaminonaphthalene gave with simultaneous elimination of formic acid 1,5-diaryl-3-[2-(naphtho[2,3-d]imidazol-2-yl)]-formazans (2a-2f). The oxidative cyclization of formazans (2a-2f) was performed by the action of lead(IV)tetraacetate in chloroform solution. With respect to the fact that products are nearly colourless crystalline compounds and there is not characteristic band for arylazo group in the visible part of UV-VIS spectrum, it is possible to exclude the structure expressed by formula (6), so that studied compounds are 2,3-diaryl-5-(naphtho[2,3- $d$ ] imidazol-2-yl)tetrazolium chlorides (4a-4f). Compounds (4) form corresponding hydrates by the crystallization from water. The chlorides were also transformed to the corresponding picrates (5a-5f). Reductive splitting of compounds (2a-2f) with $\mathrm{H}_{2} \mathrm{~S}$ proceeds smoothly to the corresponding $N^{\prime}$-aryl-naphtho[2,3- $d$ ]imidazole-2-carbohydrazonamide (3a-3f).

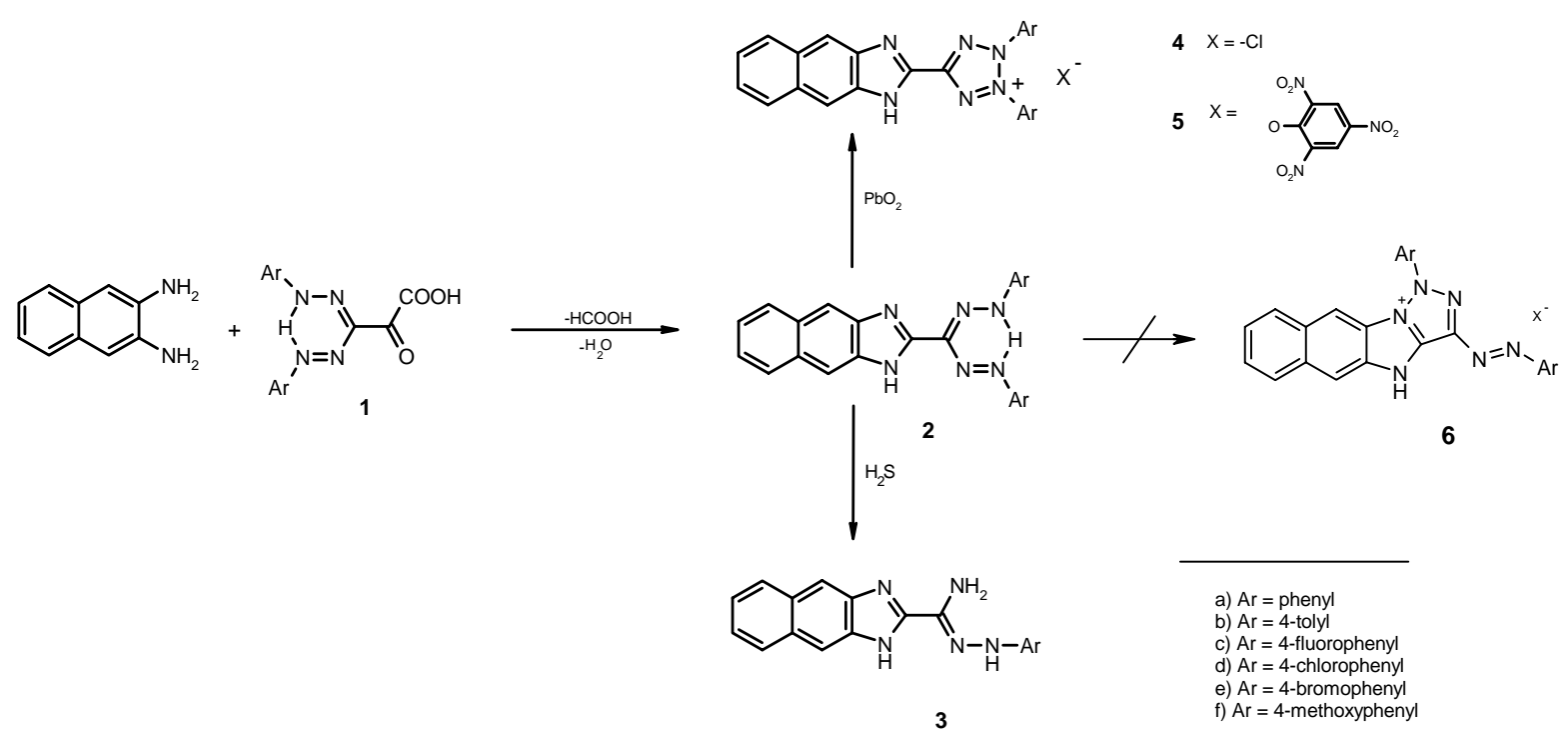




\section{Experimental Section}

1,5-Diaryl-3-(naphtho[2,3-d]imidazol-2-yl)formazans (2a-2f). General procedure. The mixture of formazylglyoxylic acid ${ }^{6}$ (1a-1f) $(1.00 \mathrm{mmol})$ and 2,3-diaminonaphtalene (158.2 mg; $1.00 \mathrm{mmol})$ refluxed for $5 \mathrm{~min}$ in ethanol $(20.0 \mathrm{ml})$. After cooling to $20^{\circ} \mathrm{C}$, the red crystalline compound was filtered off, washed with water and dried. It was purified by recrystallization from ethanol.

1,5-(Diphenyl)-3-(naphtho[2,3-d]imidazol-2-yl)formazan (2a). Red crystals, yield 95.0 \%, mp 242-243 ${ }^{\circ} \mathrm{C}, \mathrm{M} / \mathrm{S}$ (ESI, m/z (rel. \%)): 391.3 (100) (M+1) ${ }^{+}$, IR (KBr) v: 3269, 3061, 1598, 1554, 1478, 1417, 1376, 1336, 1285, 1231, 1168, 1098, 849, 765, 730, 537. UV: $\lambda \max 323 \mathrm{~nm}(\log \varepsilon$ 5.08), $440 \mathrm{~nm}\left(\log \varepsilon\right.$ 5.04). ${ }^{1} \mathrm{H}$ NMR (DMSO) $\delta: 7.38\left(\mathrm{t}, 2 \mathrm{H}, \mathrm{J}=7.5\right.$, ArH); 7.43(dd, 2H, $\mathrm{J}_{1}=6.3$, $\left.\mathrm{J}_{2}=3.0, \mathrm{ArH}\right) ; 7.58(\mathrm{t}, 4 \mathrm{H}, \mathrm{J}=8.1, \mathrm{ArH}) ; 7.94(\mathrm{~d}, 4 \mathrm{H}, \mathrm{J}=7.5, \mathrm{ArH}) ; 8.06\left(\mathrm{dd}, 2 \mathrm{H}, \mathrm{J}_{1}=6.3, \mathrm{~J}_{2}=3.0\right.$, ArH); 8.34(s, 2H, ArH); 10.21(s, $1 \mathrm{H}, \mathrm{NH}) ; 13.97(\mathrm{~s}, 1 \mathrm{H}, \mathrm{NH})$. Anal. Calcd. For $\mathrm{C}_{24} \mathrm{H}_{18} \mathrm{~N}_{6}$ (390.45): C, 73.83; H, 4.65; N, 21.52. Found: C, 73.78; H, 4.60; N, 21.62.

1,5-[Di-(4-tolyl)]-3-(naphtho[2,3-d]imidazol-2-yl)formazan (2b). Red crystals, yield 89.9 \%, mp 276-277 ${ }^{\circ} \mathrm{C}, \mathrm{M} / \mathrm{S}$ (ESI, m/z (rel. \%)): 419.5 (100) (M+1) ${ }^{+}$, IR (KBr) v: 3290, 3217, 3027, 1593, 1556, 1486, 1429, 1283, 1239, 815, 505. UV: $\lambda \max 330 \mathrm{~nm}(\log \varepsilon$ 4.43), $457 \mathrm{~nm}(\log \varepsilon$ 4.31). ${ }^{1} \mathrm{H}$ NMR (DMSO) $\delta: 2.39\left(\mathrm{~s}, 6 \mathrm{H}, \mathrm{CH}_{3}\right) ; 7.38(\mathrm{~d}, 4 \mathrm{H}, \mathrm{J}=8.4, \mathrm{ArH}) ; 7.43\left(\mathrm{dd}, 2 \mathrm{H}, \mathrm{J}_{1}=6.3\right.$, $\mathrm{J}_{2}=2.9$, ArH); 7.83(m, 4H, ArH); 8.06(dd, 2H, $\mathrm{J}_{1}=6.3, \mathrm{~J}_{2}=2.9$, ArH); 8.33(s, 2H, ArH); 10.21(s, $1 \mathrm{H}, \mathrm{NH}) ; 13.94(\mathrm{~s}, 1 \mathrm{H}, \mathrm{NH})$. Anal. Calcd. For $\mathrm{C}_{26} \mathrm{H}_{22} \mathrm{~N}_{6}$ (418.5): C, 74.62; H, 5.30; N, 20.08. Found: C, 74.70; H, 5.20; N, 20.10.

1,5-[Di-(4-fluorophenyl)]-3-(naphtho[2,3-d]imidazol-2-yl)formazan (2c). Red crystals, yield $87.0 \%$, mp 280-281 ${ }^{\circ} \mathrm{C}, \mathrm{M} / \mathrm{S}$ (ESI, m/z (rel. \%)): 427.1 (100) (M+1) ${ }^{+}$, IR (KBr) v: 3316, 3261, 1591, 1565, 1491, 1434, 1372, 1284, 1222, 1171, 1098, 829, 757, 508. UV: $\lambda \max 326 \mathrm{~nm}(\log \varepsilon$ 4.39), $436 \mathrm{~nm}\left(\log \varepsilon\right.$ 4.30). ${ }^{1} \mathrm{H}$ NMR (DMSO) $\delta: ~ 7.42(\mathrm{~m}, 6 \mathrm{H}, \mathrm{ArH}) ; 7.96(\mathrm{~m}, 4 \mathrm{H}, \mathrm{ArH}) ; 8.06(\mathrm{dd}$, $\left.2 \mathrm{H}, \mathrm{J}_{1}=6.3, \mathrm{~J}_{2}=3.6, \mathrm{ArH}\right) ; 8.31(\mathrm{~s}, 2 \mathrm{H}, \mathrm{ArH}) ; 10.23(\mathrm{~s}, 1 \mathrm{H}, \mathrm{NH}) ; 13.99(\mathrm{~s}, 1 \mathrm{H}, \mathrm{NH})$. Anal. Calcd. For $\mathrm{C}_{24} \mathrm{H}_{16} \mathrm{~N}_{6} \mathrm{~F}_{2}$ (426.43): C, 67.60; H, 3.78; N, 19.71. Found: C, 67.57; H, 3.82; N, 19.80.

1,5-[Di-(4-chlorophenyl)]-3-(naphtho[2,3-d]imidazol-2-yl)formazan (2d). Red crystals, yield 91.4 \%, mp 284-285 ${ }^{\circ} \mathrm{C}, \mathrm{M} / \mathrm{S}$ (ESI, m/z (rel. \%)): 459.2 (100) (M+1) ${ }^{+}$, IR (KBr) v: 3315, 1550, 1475, 1291, 1229, 1092, 831, 742, 612, 501. UV: $\lambda \max 325 \mathrm{~nm}(\log \varepsilon$ 4.42), $440 \mathrm{~nm}(\log \varepsilon$ 4.37). ${ }^{1} \mathrm{H}$ NMR (DMSO) $\delta: ~ 7.42\left(\mathrm{dd}, 2 \mathrm{H}, \mathrm{J}_{1}=6.3, \mathrm{~J}_{2}=3.0\right.$, ArH); 7.63(d, 4H, J=8.7, ArH); 7.94(d, $4 \mathrm{H}, \mathrm{J}=8.7, \mathrm{ArH}$ ); 8.05(dd, 2H, J $\left.=6.3, \mathrm{~J}_{2}=3.0, \mathrm{ArH}\right)$; 8.31(s, 2H, ArH); 13.93(s, $\left.1 \mathrm{H}, \mathrm{NH}\right)$. Anal. Calcd. For $\mathrm{C}_{24} \mathrm{H}_{16} \mathrm{~N}_{6} \mathrm{Cl}_{2}$ (459.43): C, 62.74; H, 3.51; N, 18.29. Found: C, 62.69; H, 3.57; N, 18.22 .

1,5-[Di-(4-bromophenyl)]-3-(naphtho[2,3-d]imidazol-2-yl)formazan (2e). Red crystals, yield $86.1 \%$, mp 282-283 ${ }^{\circ} \mathrm{C}, \mathrm{M} / \mathrm{S}$ (ESI, m/z (rel. \%)): 547.1 (100) (M+1) ${ }^{+}$, IR (KBr) v: 3068, 1545, 1478, 1427, 1282, 1239, 1069, 852, 817, 566. UV: $\lambda \max 331 \mathrm{~nm}(\log \varepsilon$ 4.10), $450 \mathrm{~nm}(\log \varepsilon$ 4.24). ${ }^{1} \mathrm{H}$ NMR (DMSO) $\delta: 7.43\left(\mathrm{dd}, 2 \mathrm{H}, \mathrm{J}_{1}=6.3, \mathrm{~J}_{2}=3.0, \mathrm{ArH}\right) ; 7.75(\mathrm{~d}, 4 \mathrm{H}, \mathrm{J}=8.7$, ArH); 7.88(d, $4 \mathrm{H}, \mathrm{J}=8.7, \mathrm{ArH}) ; 8.05\left(\mathrm{dd}, 2 \mathrm{H}, \mathrm{J}_{1}=6.3, \mathrm{~J}_{2}=3.0\right.$, ArH); 8.31(s, 2H, ArH); 10.19(s, 1H, NH); 
13.98(s, 1H, NH). Anal. Calcd. For $\mathrm{C}_{24} \mathrm{H}_{16} \mathrm{~N}_{6} \mathrm{Br}_{2}$ (548.23): C, 52.58; H, 2.94; N, 15.33. Found: C, 52.53; H, 3.01; N, 15.39 .

1,5-[Di-(4-methoxyphenyl)]-3-(naphtho[2,3-d]imidazol-2-yl)formazan (2f). Red crystals, yield $94.2 \%$, mp $244-245^{\circ} \mathrm{C}, \mathrm{M} / \mathrm{S}$ (ESI, m/z (rel. \%)): 451.4 (100) (M+1) ${ }^{+}$, IR (KBr) v: 2933, 2864, 1592, 1557, 1494, 1285, 1248, 1183, 1142, 1030, 862, 827, 737, 516. UV: $\lambda$ max $336 \mathrm{~nm}$ (log $\varepsilon$ 4.42), $465 \mathrm{~nm}\left(\log \varepsilon\right.$ 4.23). ${ }^{1} \mathrm{H}$ NMR (DMSO) $\delta: 3.86\left(\mathrm{~s}, 6 \mathrm{H}, \mathrm{OCH}_{3}\right) ; 7.13(\mathrm{~d}, 4 \mathrm{H}, \mathrm{J}=9.0$, $\mathrm{ArH}) ; 7.43(\mathrm{~m}, 2 \mathrm{H}, \mathrm{ArH}) ; 7.89(\mathrm{~m}, 4 \mathrm{H}, \mathrm{ArH}) ; 8.05(\mathrm{~m}, 2 \mathrm{H}, \mathrm{ArH}) ; 8.31(\mathrm{~s}, 2 \mathrm{H}, \mathrm{ArH}) ; 10.20(\mathrm{~s}, 1 \mathrm{H}$, $\mathrm{NH})$; 13.91(s, 1H, NH). Anal. Calcd. For $\mathrm{C}_{26} \mathrm{H}_{22} \mathrm{~N}_{6} \mathrm{O}_{2}$ (450.5): C, 69.32; H, 4.92; N, 18.65. Found: C, 69.31; H, 5.01; N, 18.55.

$N^{\prime}$-Aryl-naphtho[2,3-d]-imidazole-2-carbohydrazonamide (3a-3f). General procedure: A solution of corresponding 1,5-diaryl-3-[2-(naphto[2,3-d]imidazol-2-yl)]formazane (2a2f) $(1.00 \mathrm{mmol})$ in ethanol $(50-150 \mathrm{ml})$ was saturated with $\mathrm{H}_{2} \mathrm{~S}$. The solution was allowed to stand at room temperature in closed flask with intermittent stirring for 7 days. The reaction mixture was filtered and the filtrate was evaporated in vacuo. The solid was suspended in mixture of ethanol $(5.0 \mathrm{ml})$ and water $(3.0 \mathrm{ml})$ and allowed to stand at room temperature for $2 \mathrm{~h}$. Then it was refluxed for $10 \mathrm{~min}$ and filtered hot. The filtrate was evaporated in vacuo. The product was crystallized from ethanol-water (1:1).

$\boldsymbol{N}^{\prime}$-Phenyl-naphtho[2,3-d]-imidazole-2-carbohydrazonamide (3a). Yellow crystals, yield 85.1 \%, mp $227-228{ }^{\circ} \mathrm{C}, \mathrm{M} / \mathrm{S}$ (ESI, m/z (rel. \%)): 302.3 (100) (M+1) ${ }^{+}$, IR (KBr) v: 3278, 1626, 1599, 1509, 1496, 1429, 1394, 1335, 1308, 1253, 1143, 1067, 884, 747, 695. UV: $\lambda$ max $216 \mathrm{~nm}$ (log $\varepsilon$ 3.34), $280 \mathrm{~nm}\left(\log \varepsilon\right.$ 3.02), $348 \mathrm{~nm}\left(\log \varepsilon\right.$ 3.36). ${ }^{1} \mathrm{H}$ NMR (DMSO) $\delta: 6.25\left(\mathrm{~s}, 2 \mathrm{H}, \mathrm{NH}_{2}\right)$; 7.01(m, 1H, ArH); 7.43(m, 4H, ArH); 7.58(t, 1H, J=8.1, ArH); 8.06(m, 3H, ArH); 8.32(s, 2H, ArH); 10.05(s, 1H, NH); 12.67(s, 1H, NH). Anal. Calcd. For $\mathrm{C}_{18} \mathrm{H}_{15} \mathrm{~N}_{5}$ (301.35): C, 71.74; $\mathrm{H}$, 5.02; N, 23.24. Found: C, 71.69; H, 4.98; N, 23.33.

$\boldsymbol{N}^{\prime}$-(4-Tolyl)-naphtho[2,3-d]-imidazole-2-carbohydrazonamide (3b). Yellow crystals, yield 82.0 \%, mp 254-255 ㄷ, M/S (ESI, m/z (rel. \%)): 316.3 (100) (M+1) ${ }^{+}$, IR (KBr) v: 3154, 3037, 1638, 1612, 1512, 1437, 1333, 1249, 1172, 812, 715, 631. UV: UV: $\lambda$ max 208 nm (log $\varepsilon$ 3.48), $296 \mathrm{~nm}\left(\log \varepsilon\right.$ 3.18), $352 \mathrm{~nm}\left(\log \varepsilon\right.$ 3.21). ${ }^{1} \mathrm{H}$ NMR (DMSO) $\delta: 2.40\left(\mathrm{~s}, 3 \mathrm{H}, \mathrm{CH}_{3}\right) ; 6.15(\mathrm{~s}, 2 \mathrm{H}$, $\left.\mathrm{NH}_{2}\right) ; 7.19(\mathrm{~m}, 4 \mathrm{H}, \mathrm{ArH}) ; 7.50(\mathrm{~m}, 4 \mathrm{H}, \mathrm{ArH}) ; 8.28(\mathrm{~s}, 2 \mathrm{H}, \mathrm{ArH}) ; 10.01(\mathrm{~s}, 1 \mathrm{H}, \mathrm{NH}) ; 12.84(\mathrm{~s}, 1 \mathrm{H}$, $\mathrm{NH}$ ). Anal. Calcd. For $\mathrm{C}_{19} \mathrm{H}_{17} \mathrm{~N}_{5}$ (315.38): C, 72.36; H, 5.43; N, 22.21. Found: C, 72.29; H, 5.37; $\mathrm{N}, 22.34$.

$N^{\prime}$-(4-Fluorophenyl)-naphtho[2,3-d]-imidazole-2-carbohydrazonamide (3c). Yellow crystals, yield $87.9 \%$, mp $264-265{ }^{\circ} \mathrm{C}, \mathrm{M} / \mathrm{S}$ (ESI, m/z (rel. \%)): 320.2 (100) (M+1) ${ }^{+}$, IR (KBr) v: 3432, 1651, 1610, 1503, 1439, 1337, 1252, 1204, 1156, 822, 748, 583. UV: $\lambda$ max $209 \mathrm{~nm}$ (log $\varepsilon 3.32)$, $280 \mathrm{~nm}\left(\log \varepsilon\right.$ 3.00), $348 \mathrm{~nm}\left(\log \varepsilon\right.$ 3.25). ${ }^{1} \mathrm{H}$ NMR (DMSO) $\delta: 6.15\left(\mathrm{~s}, 2 \mathrm{H}, \mathrm{NH}_{2}\right) ; 7.32(\mathrm{~m}, 2 \mathrm{H}$, ArH); 7.76(m, 4H, ArH); 7.56(d, 1H, J=5.6, ArH); 7.67(d, 1H, J=5.69, ArH); 8.20(s, 2H, ArH); 10.11(s, $1 \mathrm{H}, \mathrm{NH}) ; 12.69(\mathrm{~s}, 1 \mathrm{H}, \mathrm{NH})$. Anal. Calcd. For $\mathrm{C}_{18} \mathrm{H}_{14} \mathrm{~N}_{5} \mathrm{~F}$ (319.34): C, 67.70; H, 4.42; N, 21.93. Found: C, 67.59; H, 4.45; N, 22.03.

$N^{\prime}$-(4-Chlorophenyl)-naphtho[2,3-d]-imidazole-2-carbohydrazonamide $\quad$ (3d). Yellow crystals, yield $84.3 \%$, mp 263-264 ${ }^{\circ} \mathrm{C}$, M/S (ESI, m/z (rel. \%)): 336.1 (100) (M+1) ${ }^{+}$, IR (KBr) v: 
3258, 1658, 1597, 1526, 1493, 1485, 1450, 1392, 1305, 1251, 1157, 1087, 822, 749, 588. UV: $\lambda$ max $205 \mathrm{~nm}\left(\log \varepsilon\right.$ 3.39), $277 \mathrm{~nm}\left(\log \varepsilon\right.$ 3.05), $352 \mathrm{~nm}\left(\log \varepsilon\right.$ 3.32). ${ }^{1} \mathrm{H}$ NMR (DMSO) $\delta: ~ 6.22(\mathrm{~s}$, $\left.2 \mathrm{H}, \mathrm{NH}_{2}\right) ; 7.31(\mathrm{~m}, 5 \mathrm{H}, \mathrm{ArH}) ; 7.49(\mathrm{~m}, 3 \mathrm{H}, \mathrm{ArH}) ; 8.22(\mathrm{~s}, 2 \mathrm{H}, \mathrm{ArH}) ; 12.51(\mathrm{~s}, 1 \mathrm{H}, \mathrm{NH})$. Anal. Calcd. For $\mathrm{C}_{18} \mathrm{H}_{14} \mathrm{~N}_{5} \mathrm{Cl}$ (335.84): C, 64.37; H, 4.20; N, 20.85. Found: C, 64.42; H, 4.14; N, 20.91 .

$N^{\prime}$-(4-Bromophenyl)-naphtho[2,3-d]-imidazole-2-carbohydrazonamide (3e). Yellow crystals, yield $80.2 \%$, mp $259-260{ }^{\circ} \mathrm{C}, \mathrm{M} / \mathrm{S}$ (ESI, m/z (rel. \%)): 380.2 (100) (M+1) ${ }^{+}$, IR (KBr) v: 3279, 1600, 1590, 1546, 1485, 1432, 1387, 1300, 1157, 1028, 890, 799, 478. UV: $\lambda \max 210 \mathrm{~nm}(\log \varepsilon$ 3.34), $283 \mathrm{~nm}$ (log $\varepsilon$ 3.00), $346 \mathrm{~nm}\left(\log \varepsilon\right.$ 3.22). ${ }^{1} \mathrm{H}$ NMR (DMSO) $\delta: ~ 6.27\left(\mathrm{~s}, 2 \mathrm{H}, \mathrm{NH}_{2}\right) ; 7.37(\mathrm{~m}$, 5H, ArH); 7.69(m, 3H, ArH); 8.17(s, 2H, ArH); 10.00(s, 1H, NH); 12.64(s, 1H, NH). Anal. Calcd. For $\mathrm{C}_{18} \mathrm{H}_{14} \mathrm{~N}_{5} \mathrm{Br}$ (380.24): C, 56.85; H, 3.71; N, 18.41. Found: C, 56.92; H, 3.69; N, 18.63.

$\boldsymbol{N}^{\prime}$-(4-Methoxyphenyl)-naphtho[2,3-d]-imidazole-2-carbohydrazonamide $\quad$ (3f). Yellow crystals, yield $88.1 \%$, mp 225-226 ${ }^{\circ} \mathrm{C}, \mathrm{M} / \mathrm{S}$ (ESI, m/z (rel. \%)): 332.3 (100) (M+1) ${ }^{+}$, IR (KBr) v: 3434, 3069, 3000, 1629, 1607, 1579, 1430, 1349, 1272, 1100, 715, 599. UV: $\lambda$ max $208 \mathrm{~nm}(\log$ $\varepsilon$ 3.51), $302 \mathrm{~nm}\left(\log \varepsilon\right.$ 3.16), $367 \mathrm{~nm}\left(\log \varepsilon\right.$ 3.20). ${ }^{1} \mathrm{H}$ NMR (DMSO) $\delta: ~ 3.82\left(\mathrm{~s}, 3 \mathrm{H}, \mathrm{OCH}_{3}\right.$ ); 6.13(s, 2H, NH ); 7.18(m, 4H, ArH); 7.89(m, 4H, ArH); 8.26(s, 2H, ArH); 10.18(s, 1H, NH); 12.83(s, 1H, NH). Anal. Calcd. For $\mathrm{C}_{19} \mathrm{H}_{17} \mathrm{~N}_{5} \mathrm{O}$ (331.38): C, 68.87; H, 5.17; N, 21.13. Found: C, 68.78; H, 5.06; N, 21.20.

2,3-Diaryl-5-(naphtho[2,3- $\boldsymbol{d}$ ] imidazol-2-yl)tetrazolium chlorides (4a-4f). General procedure: Lead(IV)tetraacetate (0.50 g; $1.12 \mathrm{mmol})$ was added with stirring to a solution of 1,5-diaryl-3(naphto[2,3-d]imidazol-2-yl)formazan (2a-2f) (1.00 mmol) in $\mathrm{CHCl}_{3}(50-150 \mathrm{ml})$. The solution was stirred for $3 \mathrm{~h}$ at room temperature and filtered. The filtrate was evaporated in vacuo, the residue dissolved in $\mathrm{H}_{2} \mathrm{O}(10 \mathrm{ml})$ and acidified with conc. $\mathrm{HCl}$ to $\mathrm{pH} 2$. The precipitate was filtered off and the filtrate was evaporated in vacuo. The residue was dissolved in methanol (7-10 $\mathrm{ml}$ ), filtered and evaporated again. The residue was dried in vacuum dessicator over $\mathrm{KOH}$. Compounds (4) are hygroscopic and they were transformed into less hydroscopic picrates.

2,3-Diaryl-5-(naphtho[2,3- $d$ ] imidazol-2-yl)tetrazolium picrates (5a-5f). General procedure: A solution of sodium picrate $(251.0 \mathrm{mg} ; 1.00 \mathrm{mmol})$ in $\mathrm{H}_{2} \mathrm{O}(5 \mathrm{ml})$ was added to the stirred solution of tetrazolium salt (4a-4f) $(1 \mathrm{mmol})$ in $\mathrm{H}_{2} \mathrm{O}(1-3 \mathrm{ml})$ and stirring continued for 5 minutes. The precipitated compound (5a-5f) was collected with suction and dried.

2,3-Diphenyl-5-(naphtho[2,3-d]imidazol-2-yl)tetrazolium picrates (5a). White crystals, yield 93.1 \%, mp 151-152 ${ }^{\circ} \mathrm{C}, \mathrm{M} / \mathrm{S}$ (ESI, m/z (rel. \%)): 389.3 (100) (M+1) ${ }^{+}$. UV: $\lambda \max 307 \mathrm{~nm}(\log \varepsilon$ 4.63). Anal. Calcd. For $\mathrm{C}_{30} \mathrm{H}_{19} \mathrm{~N}_{9} \mathrm{O}_{7}$ (617.54): C, 58.35; H, 3.10; N, 20.41. Found: C, 58.29; H, 3.22; N, 20.43.

2,3-[Di-(4-tolyl)]-5-(naphtho[2,3-d]imidazol-2-yl)tetrazolium picrates (5b). White crystals, yield $92.0 \%$, mp 183-184 ${ }^{\circ} \mathrm{C}, \mathrm{M} / \mathrm{S}$ (ESI, m/z (rel. \%)): 417.2 (100) (M+1) ${ }^{+}$. UV: $\lambda \max 313 \mathrm{~nm}$ (log $\varepsilon$ 4.71). Anal. Calcd. For $\mathrm{C}_{32} \mathrm{H}_{23} \mathrm{~N}_{9} \mathrm{O}_{7}$ (645.59): C, 59.54; H, 3.59; N, 19.53. Found: C, 59.53; H, 3.68; N, 19.47. 
2,3-[Di-(4-fluorophenyl)]-5-(naphtho[2,3-d]imidazol-2-yl)tetrazolium picrates (5c). White crystals, yield $91.9 \%$, mp 178-179 ${ }^{\circ} \mathrm{C}, \mathrm{M} / \mathrm{S}$ (ESI, m/z (rel. \%)): 425.1 (100) (M+1) ${ }^{+}$. UV: $\lambda$ max $309 \mathrm{~nm}\left(\log \varepsilon\right.$ 4.71). Anal. Calcd. For $\mathrm{C}_{30} \mathrm{H}_{17} \mathrm{~N}_{9} \mathrm{O}_{7} \mathrm{~F}_{2}$ (653.52): C, 55.14; H, 2.62; N, 19.29. Found: C, 55.21; H, 2.55; N, 19.22 .

2,3-[Di-(4-chlorophenyl)]-5-(naphtho[2,3-d]imidazol-2-yl)tetrazolium picrates (5d). White crystals, yield $86.9 \%$, mp 194-195 ${ }^{\circ} \mathrm{C}, \mathrm{M} / \mathrm{S}$ (ESI, m/z (rel. \%)): 457.1 (100) (M+1) ${ }^{+}$. UV: $\lambda$ max $312 \mathrm{~nm}$ (log $\varepsilon$ 4.63). Anal. Calcd. For $\mathrm{C}_{30} \mathrm{H}_{17} \mathrm{~N}_{9} \mathrm{O}_{7} \mathrm{Cl}_{2}$ (686.52): C, 52.49; H, 2.50; N, 18.36. Found: C, 52.51; H, 2.46; N, 18.30 .

2,3-[Di-(4-bromophenyl)]-5-(naphtho[2,3-d]imidazol-2-yl)tetrazolium picrates (5e). White crystals, yield $92.6 \%$, mp 174-175 ${ }^{\circ} \mathrm{C}, \mathrm{M} / \mathrm{S}$ (ESI, m/z (rel. \%)): 545.3 (100) (M+1) ${ }^{+}$. UV: $\lambda$ max $310 \mathrm{~nm}$ ( $\log \varepsilon$ 4.63). Anal. Calcd. For $\mathrm{C}_{30} \mathrm{H}_{17} \mathrm{~N}_{9} \mathrm{O}_{7} \mathrm{Br}_{2}$ (775.32): C, 46.47; H, 2.21; N, 16.26 . Found: C, 46.44; H, 2.27; N, 16.16.

2,3-[Di-(4-methoxyphenyl)]-5-(naphtho[2,3-d]imidazol-2-yl)tetrazolium picrates (5f). White crystals, yield $88.1 \%$, mp 165-166 ${ }^{\circ} \mathrm{C}, \mathrm{M} / \mathrm{S}$ (ESI, m/z (rel. \%)): 449.2 (100) (M+1) ${ }^{+}$. UV: $\lambda$ max $307 \mathrm{~nm}$ ( $\log \varepsilon$ 4.49). Anal. Calcd. For $\mathrm{C}_{32} \mathrm{H}_{23} \mathrm{~N}_{9} \mathrm{O}_{9}$ (677.59): C, 56.72; H, 3.42; N, 18.60. Found: C, 56.78; H, 3.37; N, 18.65.

Melting points (Boetius) are not corrected. Infrared spectra were recorded as potassium bromide disks and scanned on an ATI Unicam Genesis FTIR instrument. MS spectra were recorded on ZAB-EQ (VG Analytical Ltd., England). The NMR spectra were recorded in DMSO- $\mathrm{d}_{6}$ solutions on a Bruker AMX-300 spectrometer $(300 \mathrm{MHz})$ with TMS as internal standard. Elemental analyses were performed using an EA Elemental Analyzer (Fison Instrument).

\section{Acknowledgments}

We are grateful to the Ministry of Education, Youth and Sport of the Czech Republic, for the grant MSM6198959216.

\section{References}

1. For previous paper see ref. 7

2. Hinsberg, O.: Liebigs Ann. Chem. 1896, 292, 245.

3. Simpson, J. C. E.:Condensed pyridazine and pyrazine rings Weissberger, A. E., ed. Interscience New York (1953).

4. Platt, Y. T.: The Quinoxalines - In Heterocyclic Compounds Vol.6, Chapter 10, R.C. Elderfield, R. C. Ed, Wiley: New York, 1956.

5. Morrison, D. C.: J. Am. Chem. Soc. 1954, 76, 4483.

6. Wiedermannová I., Slouka J., Lemr K.: Heterocyclic Commun. 2002, 8, 479.

7. Fryšová I., Slouka J., Gucký T.: Arkivoc 2005, (xv), 1-3.

8. Zemplén G., Mester L., Messmer A.A.: Chem. Ber. 1953, 86, 697. 